\title{
Spiros Simitis \\ Die Loi le Chapelier: Bemerkungen zur Geschichte und möglichen Wiederentdeckung des Individuums
}

\author{
Für eine Wahrnelumung von Recht als Reche gehe es um

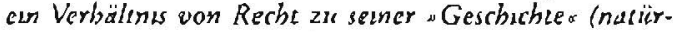 \\ lich auch in je verwirklichten . Gescllschaften $\times$ ) wie isy \\ jeweils zeugenössischen "Gesellschafta fwrederum: and \\ ithrer Geschuchte). \\ R. Wiechölter, KJ 21 (1988) 407
}

1.

Die Diagnose fällt ebenso klar wie lapidar aus: das Individuum, so heißt es, ist längst am *Ende*, das Subjekt „ror $*$ die Zcir einer sich nur noch *dans le vide de l'homme disparua' vollzichenden Reflexion endgülrig angebrochen. Zweifel oder gar dic Erwartung, sich doch noch mic der Möglichkeit einer Rekonstruktion des Individuums auseinanderzusetzen ${ }^{2}$, werden als schliclit anachroniscisch abgetan ${ }^{3}$. Je dezidicrter aber die «Archäologie «ur Domäne aller Betrachtungen über das dekonstruierte "Subjekt" erklärt wird, desto unauswcichlicher crscheinc auch die radikale Revision aller das "Projekt « der Moderne kennzeichnenden rechtlichen Atrribute: Der einst für selbstverständlich gehaltenc Konnex zwischen den Grundrechten und der Anerkennung des Individuums zerbricht; die Grundrechte garancieren nicht mehr die individuelle Autonomie, sie sichem das Netzwerk der Organisarionen $\alpha$ ab, gewährleisten dessen Funktionsfähigkeit."

Die Gcgenposition ist wohl selren wieder so eindrucksvoll formuliert worden, wie in jener Debarre der Französischen Nationalversammlung, die in der Nachc des 4. August 1789 begann und mit der Verabschiedung der Loi le Chapelier am 14. Juni 1791 endere. wIl n'y a plus de corporation dans l'ćcar; il n'y a plus que l'intérêt particulier de chaque individu, et l'intérêt généralass, stellte der Berichecrstatter, der Abgeordncte Le Chapelier, fest ${ }^{6}$ und fügte hinzu: sIl n'est permis à personne d'inspirer aux citoyens un intérêz intermédire, de les séparer de la chose publique par un exprit de corporations. «7 Konscquenterweise heißı es dann in Arr. I des Gesetzes: "L'anéantissement de toutes espèces de corporations de citoyens de mème écat et profession, étant l'une des bases fondamentales de la Consritution française, il est défendu de les rétablir de fait, sous quelque prétexte et sous quelque forme que ce soit. $\ll^{8}$

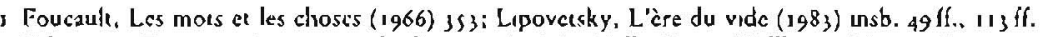

2 Vgl. etwa Tourainc, Le retour de l'acteur (1984); Heller/Sosna/Wellbcry (Hrsg,), Reconstrucang individualism (1986).

3 Stau aller Baudrillard, Cool Memorırs (1987), Magazıne lutéraıre Heír 364 (A pril 1989) igff.

4 Ladeur, KJ 1986, 197 If. und 1987, 1 50 fl. (1,6).

s Es gibi kene Korporatıon mehe im Staal; cs gibt nur noch das parikularc lnteresse jedes cin'zelnen und das Allgemonniteressc.

6 Archives Parlementaires, lère sènc, $27(1887) 210$.

7 Es ist niemandem erlaubt, den Bürgern ein intermediäres Inccressc nahezulegen, sic vom Gemesnwesen durch einen Korporsuonsgeise zu trennen.*

\$ weil die Vernuchiung aller Aren von Korporacionen der Bürger desselben Standes und Berufes zu den wucheigseen Grundlagen der französischen Verfassung zàhlt, ist es verboten. die Kopporawoncn der 
Kaum ein anderes Gesecz hat wie die Loi le Chapelier nicht nur die wechselnden Phasen der Revolution unverändert überstanden, sondern auch unangefochten bis rief in das 19.Jahrhundert hinein weitergegolten. Und von kaum einer anderen legislativen Entscheidung der Nationalversammlung läßs sich sagen, daß sie, wie die Loi le Chapclier, bis heute zu den gleichsam selbstverständlichen Orientierungspunkten weiter Teile der juristischen Literatur gehört. $\$$ Nur noch vereinzelt wird freilich in der Loi le Chapelier der konstitutive Akt eines radikalen Individualismus gesehen. ${ }^{\circ}$ Weitaus geläufiger ist es vielmehr, gerade den schon erwähnten Art. I des Gesetzes als die „Geburtsurkunde des Prolecariats a auszugeben." Gane in diesem Sinn hat schon Karl Marx das Gesctz keineswegs nur zum Anlaß genommen, um Robespierre zu kritisieren,' ${ }^{2}$ vielmehr die Loi le Chapelier als manifesten Beweis eines "bürgerlichen Staatsstreichs« bezeichnet, dcr einzig und allein das Ziel verfolgc habe den Konkurrenzkampf zwischen Kapital und Arbeir staatspolizeilich innerhalb dem Kapital bequemer Schranken (einzuzwängen) '" , cin Urteil, dessen Richrigkeit, wic Jürgen Kuczynski mcinc ${ }^{14}$, durch den "sofortigen * und menergischen* Protest Marats und damir eines der wichtigsten Repräsentanten der Linken bestätigt würde.

Die vielen, eindeutig negativen Bemerkungen haben einen durchaus verständlichen Hintergrund. Der ursprünglich breit angelegıc Anwendungsbereich des Gesetzes wurde schon bald nach der Verabschiedung gezielt eingeenge. Von den "Korporationen" war kaum noch die Rede, dafür um so mehr von den verschiedenen Versuchen der Arbeitnchmer, sich gewerkschaftlich zu organisieren. Das in Art. I formulierte allgemeine Korporationsverbot verwandelte sich so zunehmend in cine ausschließlich auf die Gewerkschafren anwendbare Regelung. Die Loi lo Chapelier reihte sich damit in die lange Liste jener Gesetze ein, dice wie etwa das dänische Dekret vom März 1800, die britischen Combination Acts von 1799 und 1800 oder die württembergische GewO von 136 den Arbeitnehmern jegliche Koalitionsmöglichkeit absprachen. Das weitere Schicksal des Geserzes bestätigı dic veränderte Sichc. Die Loi le Chapelier wurde zusammen mir dem einschlägigen Abschnitt des Strafgesetzbuches erst aufgehoben," als sich der Gesetzgcber, nicht zuletzt unter dem Eindruck der wachsenden Schwierigkeiten, das Verbot durclizuserzen, für eine Polizik entschied, die zwanzig Jalure früher, am 22. April 1864 , Ollivier in seinen an die Adresse des Corps Législatif gerichreten Bemerkungen zur Encwicklung der Koalitionsgeserzgebung so zusammengefaßt hatte: „Avec le temps la libercé des coalitions tuera la grève. . $^{16}$ Genaugenommen nur cine cbenso kurze wie prägnante Wiedergabe jener Überlegungen, die John Stuart Mill schon 1848 formuliert hatte:

Sache nach wiederberzustellen, uneer welchem Vorwand und in weicher Farm dies auch geschehen mag. *

9 Vgl. erwa Durand/Jaussand, Tratc du drone du travail I (1947) 68 f.; Javillier, Drout du travail (2. Aufl. 198r) 95; Hueck/Nipperdey, Lehrbuch des Arberusteches II, (7. Aufl. 1967) 11 ; Veneziand, in Hepple (Hrsg.). The Makung of Labour Law in Europe (1986) ss; Jacobs, ebd. 197 l.

10 Vgl. eewa Meynaud, Nouvelles cindes sur les groupes de pression en France (1962) 348; Bouvier-A jam/ Mury, Les elasses sociales en France I (1963) 360; Kahn-Freund, Anmerkung in: Renncr, Dic Rechesunsutute des Pnvaurechis und ihre soziale Funkuon (196s) 226.

1 Daujat, in Les écapes de la législation corpotalive en France (19-42) $22 \mathrm{~g}$.

12 In ennem an Engels gerichictem Bnef vom 30. Januar 1865 , Karl Marx/Fnedrich Engels, Werke 31 (1965) 48.

1) Das Kapical Bd. 1, Mard/Engels, Werke $23(1962) 769 f$.

14 Die Geschichie der Lage der Arbeiter unter dem Kapralismus. V1 1: Dir Geschichie der Lage der Arbester in Frankreich von 1789 bis in die Gegenwar, 1789 bis $1 \$ 30$ (195s) is 4

is Cade pénal an. 414-4 16: Lou relative i la críation des syndicats professionncls vom 21 . Mark i 884 , dazu Dolléans/Dehave, Historre du travail en France I (1953) yssif.

16 Dalloz Pérıodique 1864, 66. 
"Experience of strikes has been the best teacher of the labouring classes on the subject of the relation between the wages and the demand and supply of labour: and it is most important that this course of instruction should not be disturbed. " $^{\prime 7}$ So erklärlich die Kritik an der Loi le Chapelier auch sein mag, so wenig darf sie dazu führen, das Gesetz lediglich unter dern Aspekt seiner späteren Instrumentalisierung in der Auseinandersetzung mit ciner ihre Interessen zunehmend dezidierter wahrnehmenden Arbciterschaft zu betrachten. ${ }^{18}$ Wer diesen Weg beschreiter, führ die Diskussion ausschließlich aus der Perspektive einer nacheräglich eingetreconen Encwicklung, interessien sich mithin gar nicht erst für die sich in der Forderung nach einem generellen Korporationverbot artikulierenden Vorstellungen, sondern projiziert auf die legislative Entscheidung Erwartungen, dic crst später entstanden oder zumindest voll zur Geltung gelangt sind. Wenn deshalb die spezifische, der radikalen Absage an die Etablienung und Verselbständigung intermediärer Gewalten beigemessene Bedeurang für die Konsciruierung einer strikt am Individuum orientierten Gesellschaft wirklich verstanden werden soll, gilt es, genau umgekehrt zu verfahren, also vor jeder wciteren Überlegung den legislativen Entscheidungsprozeß nachzuzeichnen.

2.

Isaac René Guy le Chapelier war weder ein unbedeutender Hinterbänkler,' ${ }^{19}$ noch ist lediglich zu vermerken, daß er keine drei Jahre nach der Verabschiedung des nach ihm benannten Gesetzes, am 22. April 1794, gouillociniert wurde. ${ }^{20}$ Le Chapelier, am 12. Juni 7754 in Rennes geboren, arbeitete dort als Rechtsanwalt und wurde im Frühjahr 1789, ebenfalls in Rennes, vom Dritcen Stand in die Generalsände gewählt. Er gründete den Bretonischen Club, der sich in Paris, nach dem Umzug der Nationalversammlung, als "Société des Amis de la Constitution « bezeichnete, abcr in die Geschichte unter dem Namen seines Tagungsortes, des Jakobinerklosters in der Rue Saint-Honoré eingegangen ist. Le Chapelier gehörte zu den profiliertesten Befünworcrn der Abschaffung des Adels und der Einführung von Geschworenengerichten. Kurz nach der Fluchı des Königs und seiner Verhaftung am 21. Juni $179 \mathrm{Y}$ in Varennes verließ Le Chapelier den Jakobinerclub und trat den Feuillants bei, die im Julj 1791 von einer Gruppe gemäßigter Jakobiner gegründet worden war, die nicht zuletzt aus Arhängern La Fayettes bestand. Nach dem Ablauf seines Mandats kehrte er nach Rennes zurück, wurde aber sehr bald immer wieder offen des "Moderancismusa bexichrigt und, schlimmer noch, der Spionage für England. Beides führte schließlich zu seiner Verhaftung und Vcrurteilung.

Bleibe cin gerade für die Geschichre des Geserzes wichriges biographisches Detail: Am 3. Auguse 1789 wurde Le Chapelier nach dem Verzicht von Thourec zum Präsidenten der Nationalversammlung gervählt." Er war es deshalb, der die Sitzung in jener Nacht des 4. auf den S. August 1788 leitete, in der sämtliche Privilegien

17 Principles of politucal coonomy, with some of their applications to socid philosophy (7. Aufl., 1871. Neudruck 1965) 932.

18 Vgl. elwa Helmich, Arbeitskümpie in Frankreach (1992) insb. 19ff.: Jacobs, 1. a. O. (Anm, 9) 198

19 Der Monteur verzeıchnct für 1990119 Wortmeldungen van Mirsbiau, 43 van Dupor, 46 von Pǘlion,

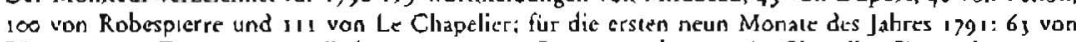
Périon, 7 I von Dupor, 95 von Robespierre, 71 von Barrave und go von Le Chapelier. Zur parlamentanschen Aktıvitit Le Chapelicrs vgl. auch Dumont, Souvenırs sur Mirabcau ce sur les deux premieres Assemblées législatives (hrsg. von J. Bénciruy' 1951) 266; FureuHalćvi. Orateurs de la Rivolueson françuse I (1939) 1\}\}s.

20 Kuczyngki, Lage is4.

$21 \mathrm{Zu}$ den Einzelheteen sciner Wahl und ru den ihr varausgegangenen Auseinanderseczungen: Arebives Parlementases, Lère séric, 8 (1875) 331, 335, sowic zulcize Bridin, Sicyds (1986) 132 
verworfen und die Grundlagen für eine egalitäre, auf der Souveränitär des Individuums beruhenden Gesellschaft gelegt wurden. Le Chapelier war es auch, der dem König die das Ende der Fcudalgeseilschaft besiegelnden und den Beginn einer neuen Epoche ankündigenden Beschlüsse der Nationalversammlung überbrachtc.

Le Chapelier gehörte mishin zu jenen Abgeordneten, dic mic ihren Vorschlägen und Überlegungen die Debarte über die Grundvoraussetzungen einer individualistisch orientierten Gesellschaft entscheidend mitgeprägt haben. Für sie stand spätestens seir den Vorgesprächen am Abend des 3. August fest, daß die Nationalversammlung nicht umhin könnte, nun endlich den Schritt zu gehen, den sic am Tag darauf nach den überraschenden Anträgen des Vicomte de Noailles und des Herzogs von Aiguillon tatsächlich vollyogen hat: $\nu$ détruire tous les privilèges des classes, des provinces, des villes er des corporarions $\alpha^{22}$. Der Text der Beschlüsse liest sich freilich anders, und zwar auch in der endgültigen am 11 . August verabschiederen Fassung. Dic Korporationen blciben unerwähnt, statt dessen ist lediglich von den "communautésu die Rede. ${ }^{23}$ Gemcint waren damit aber ausschließlich, wic nicht zuletzr die späteren Debatten immer wieder bestátigt häben, Einwohnergemeinschaften, nicht jedoch Berufsorganisationen gleichviel welcher Art.24

Auch in den nächsten Tagen, als die Sprache schärfer wurde und die Formulierungen sehr viel cnrschicdener ausfielen, finder sich kcin Hinweis auf die Korporation. Die Nationalversammlung erklärte zwar auf Antrag des Abgcordneten Duport das Fcudalregime unmißverständlich und uneingeschränkt für abgeschaffr, ging dabei aber nicht auf die Frage ein, ob damit zugleich das Ende aller intermediärcn Gewalten gemeint sei. ${ }^{\prime \prime}$ Erst am 26. August äußcrte sie sich direkt zu den Korporawionen, allerdings nichr in der Erklärung der Menschen-und Bürgerrechre, sondern in den glcichzeitig verabschiederen Anwendungsrichtlinicn. ${ }^{26}$ Dort wird zunächst festgehalten: „Il n'y a plus pour aucune partie de la nation, ni pour aucun individu, aucun privilège ... «, um dann forczufahren: „Il n'y a plus ni jurandes, ni corporations de professions, arts et mériers." Die Folgen sind in der, freilich genauso aligemein gebaltenen Aulzähiung der "Dispositions fondamentales garantics par la Conscitution " angedeutet, ${ }^{17}$ zum cinen der freic und ungehinderte, einzig durch Begabung und Tugend eingeschränktc Zugang zu allen Arbeits- und Berufsmöglichkeiten, zum anderen die Verpflichtung, ein an die Adresse jedes einzelnen gerichtetes, gleichermaßen verbindliches Zivilgeserzbuch zu schaffen. Die Absage an jegliches, welcher intermediären Gewalt auch immer vorbehaltenes Regelungsvorrecht schlägr so in jenes Regclungsprinzip um, das allein einer Gesellschaft freier, gleicher und souverän über die eigenen Interessen bestimmender Individuen angemessen ist, in den Grundsacz der Verragsfreiheit, den festzuschreiben, die cigencliche und wichtigste Aufgabe der geforderten Kodifikation ist.

Kurzum, so sehr die Schwerpunkte im Verlauf der im August 1789 geführten Debatten gewechselt haben und so unterschiedlich die Formulierungen in den einzelnen Beschlüssen ausgefallen sein mögen, so wenig läßr sich bestreiten, da $\bigcap_{3}$ sich die Nationalversammlung scit jener Nachi des 4 . August für einen radikalen, mit der Existenz intermediärer Gewalten unvereinbaren Individualismus ausgesprochen

\footnotetext{
22 Archives Parlementarres a. a. O., $343 \mathrm{ff}$, sowie Hirsch, $\mathrm{L}_{3} \mathrm{Nuut}$ du 1-Aoút (1978); Furet, in: Fured) Ozouf. Dicuonnatre critıque de la Révolution françase (1988) 126 ir.

23 Vgl. Lous Blanc. Hisıorre de la Révoluuon françasse 3 (Neuauflage 1878) 269.

24 Blanc a. a. O. Bouvicr-A jam, Histoıre du eravail en France des origınes à la Révolution (1957) 690.

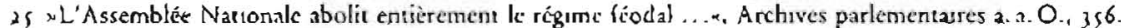

Mit einer last worılichen Wriedergabe dieses Satzes beginne auch das am 11. August 1789 verabschicdetc

Dekrce uber die Abschallung der Feudalrechie. Archives Parlementares a. a. O.. 397.

26 Abgedruckt bei Blanc, Histairs, a. .. O., $319 \mathrm{ff}$.

27 Ebcrifalls abgculruck, bes Blanc, Historre, a. a. O.
} 
hat. Wie konsequent an dieser Position festgehalten wurde, zeigte sich cin Jahr später bei den Berarungen über die Versammlungs- und Vercinigungsfreiheit. ${ }^{18}$ Zwar bestand durchaus Konscns über das Recht aller Bürger, sich um der cigenen Meinungsbildung willen jederzeit frej zu versammeln und jede von ihnen gewünschte Vereinigung zu bilden. Was immer aber zugunsten bcider Freiheiten gesagt wurde, stand durchweg unter dem von Sieyès ${ }^{29}$ und Condorcet im Hinblick auf die konsuitutionelle Anerkennung der Mensclien- und Bürgerrechte formuliertcn Vorbehalt: "Il n'existe dans l'Etar que des ciroyens divisés par cantons, et des hommes chargés par les citoyens des fonctions publiques; toute association est donc nécessairement une association privée qui doit ètre libre, mais qui ne peut avoir droit de se donner une existence comme corps. "so Konsequenterweise stellte deshalb Marar' dem jedem cinzelnen zustehenden und nur von ihm auszuübenden Recht, alle staatlichc Aktivität zu überwachen, dic spezifische Aufgabe der politischen Clubs gegenüber, "d'éclairer les esprits, de propager les lumières et le patriotisme“. Die Grenze zwischen der ebenso legitimen wie legalen Vereinsbildung cincrseits und der illegitimen und illegalen Konsticuicrung, intermediäre Belange begünsrigender und so die Fähigkeit sowie die Chancen des Individuums, seine Interessen zu erkennen und zu vertreten, zuriefst gefährdender „facrions « andererseirs ist damit einmal mehr klar gezogen. Der Weg zum Individuum ist nicht mit Zwischenstationen versehen. Eben deshalb erscheint es auch inakzeprabel, Alternativen zur individuellen Meinungs- und Entscheidungsbildung hinzunchmen, jedenfalls solange es um all jene Entscheidungsprozesse geht, von dcnen die weiterc gesellschafrliche, politische und ökonomische Entwicklung abhängt. Die Vereinigungslreiheit darf, so gesehen, niche zur Vorstufe einer Rekonstrukcion intermediärer Gewalten werden, sie ist viclmehr nur als Mittel zur besseren Vorbereitung der individucllen Akrivität tolerabel.

Verständlicherweise konnte sich daher die Nationalversammlung, als kurz darauf die Zulässigkeit von Korporationen erncut zur Debatte stand, auf keine Konzessionen einlassen, ohne sich selbst zu desavouieren. Vordergründig ging es um ganz andere Fragen. Im Auftrag des für die öffenclichen Abgaben zuständigen Ausschusses hatte der Abgeordnetc d'Allarde am is.Februar vorgeschlagen, eine neue Abgabe vorzusehen. ${ }^{32}$ Die Freiheir des einzelnen, sich für jeden ihn interessierenden Beruf zu entscheiden, sollte nach Meinung des Ausschusses an die Verpflichtung gekoppelr werden, sich zuvor eine Erlaubnis zu besorgen und dafür eine bestimmte Gebühr zu encrichten. Der Vorwurf, einc so konzipiertc Besteuerung müßte über kurz oder lang die Wiedercntstehung in sich geschlossener, leczrlich nur auf die Durchsetzung der eigenen Belange bedachter Berufsgruppen zur Folge haben, liegt aul der Hand. D'Allarde sah dic Gefahr und beschränkte sich bei scincn Ausführungen keineswegs auf rein fiskalische Überlegungen. ${ }^{33} \mathrm{Er}$ verlangte eine gesetzliche Bestätigung der Auflösung aller Korporationen. Beides, Gebühr und Korporationsverbor, sollie zudem am gleichen Tag in Krafe rreten."

28 Da\%u Noursson, Historre de la liberté d'assocuation en France depuis 1789 \& (1920) 162.

29 Qu'est-ce que le Tiers Ecat? (Neudruck der 3. Au(hige vom Mal 1789, hrsg. von Brcdin, 1989) 120 ff., I 11 ff., 169 ff.

30 Déclaration des droits, Euvres 9 (hrsg. von A. Condorcet-O'Connor und M. F. Arago 1847-1\$49. Neuauflage 196 S Frommann Verlag) 208 fl. (•Es gibt im Stant nur durch Kantone getrennte Birger und von diesen mit öffentlichen Amtern betraute Männer. Jede Vereinigung ist deshalb noiwendigerweise einc private Verengung, die zwar fret sesn muß, aber meche das Reche baben kann, sich als Korpomion ru hansucuieren. $*$ ).

31 Amı du peuple vom 4. März 1791.

32 Archives Parlementares, lère sénic, 23 (1886) 198.

3) Archives Parlementaires, 2, a. O.. insb. 199 1.

34 Ar. 1 und 2 des vorgeschlagenen Dekreus. Arcluves Parlcmentasres, 1. .. O., 201 
Mag sein, daß d'Allardc die Erfolgschancen seines Antrags damic beträchtlich crhöht har." Dennoch wärc es falsch, seinen Bemerkungen zu den Korporationen nur scrategischen Wert beizumessen. ${ }^{36}$ Die anschlicßende Debatce zeigz, wie sehr es d'Allarde ebenso wie den anderen Abgeordneten daran lag, die ständig neu aufkommenden $Z$ weifel an der schon 1789 beschlossenen Aufhebung der Korporarionen nun endlich zu beseitigen. Die Aufmerksamkeir konzentrierte sich deshalb immer wieder auf die Korporationen. In der endgülrigen Absage an alle Versuche, sie doch noch beizubehalten, Jag nach Ansicht der Abgeordneten einer der wichtigsten Vorzüge des Geseczes. Seine Aufgabe sei es, meinte etwa der Abgeordnete Roederex ${ }^{3}$, vor allem die mit der Existenz von Korporationen unweigerlich verbundenen politischen und ökonomischen Schwicrigkeiten zu beseitigen. Politisch führten Korporationen zur Entstehung partikulärer, die Bürger gegeneinander aufbringender Intercssen, ökonomisch beeinflußten sie die Lohn- und Preisbildung zum Nachteil der Konsumenten und Arbeiter. Korporationen ließen sich deshalb weder mir der von der Vcrfassung garantierten Stellung des einzelnen, noch wie d'Allarde pointierter bemerkte, ${ }^{36}$ mit seinem ebenfalls gewährleisteten Rechc vereinbaren, seine Fähigkeit zu arbeiten, ungehindert für seine Interessen einsetzen zu können. Am 2. März 179 1 stimmte die Nationalversammlung den Vorschlägen d'Allardcs 2u. ${ }^{39}$

Die Debatte bestätigte einmal mehr: So spezifisch der jerveilige Anłaß gewesen sein mag, Reflexionen über die Korporationen erschöpfren sich niemals in Betracheungen über einzelne Berufsverbände. In Gegenteil, ganz gleich um welche Vereinigungen es auch immer ging, die Abgeordneten kehren stets zu der für sie lecztlich allein entscheidenden Frage zurück: der tendenziellen Verdrängung des Bürgers aus dem politischen, sozialen und ökonomischen Enescheidungsprozeß. Die Korporationen waren für die Abgeordneten ein Malınmal am Scheideweg zwischen einer ständisch orientierten und einer individualistisch ausgerichteten Gesellschaft. In jeder Diskussion über die Existenzberechtigung von Korporationen aktualisierce sich daher sowohl die Kritik an einer zwar zerfallenden, aber nach wie vor präsenten sozialen Ordnung, als auch die Hoffnung auf eine noch lange nichr verwirklichre neue Gesellschaft.

Anfang Mai 1791, also nur wenige Wochen vor der Verabschiedung der Loi le Chapelier, stand die Korporationsfrage erneut zur Debatze. Der Anlaß hatte freilich wiederum scheinbar nichr das Geringste damit zu tun. Am 9. Mai brachice Lc Chapelier einen Enrwurf für eine gesetzliche Regelung des Petirionsrechts ein. ${ }^{\circ} \mathrm{Die}$ Begründung läßs den Zusammenhang mit der Diskussion über die Korporationen sofort erkennen. Le Chapelier bezweifelt genausowenig wie irgendein anderer Abgcordneter das elementare Intercsse des einzclnen, sich jederzeir an das Parlament wenden zu können. Die Nationalversammlung hatte deshalb schon früher das Peritionsrecht als eines der wichtigsten Rechte des einzelnen bezeichnet. ${ }^{41}$ Daran hielt auch der ncue Encwurf fest. Lc Chapelier ging es vielmehr lediglich darum, die sich seiner Meinung nach aus der Zuordnung des Peritionsrechts zwingend ergebenden Konsequenzen zu präzisieren. ${ }^{42}$ Konkret: Weil sich dic Nationalversammlung

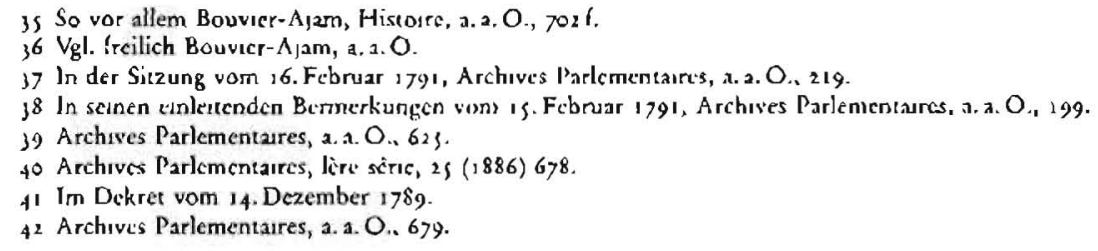


eindeutig für das Petitionsrecht als Individualrecht ausgesprochen hatte, sollte sie auch auf einer strikt individuellen Ausübung bestchen, damit aber den von den verschiedenen Verbänden und Vereinigungen immer wieder geltendgemachten Anspruch auf ein eigenes Pexitionsrechr ein und für allemal ablehnen. Jede andere Entscheidung könntc nur dazu fülıren, die individucllen Interessen zugunsten korporativer Belange zu verdrängen. So wichtig Vereinigungen und Verbände für dic individucllc Meinungsbildung auch wären, so wenig dürften sie den einzelnen substituieren. Gerade deshalb wäre ein "kollektives Petitionsrecht inakzeptabel. Sich damit abzufinden, hieße die aus souveränen Individuen bescehenden und für diese handelnden Vereinigungen und Verbände in intermediäre, den einzelnen übergeordneten Gewalten umzuwandeln.

An Widerspruch fehlte es nicht. Der Bischof von Blois zählte genauso zu den Kritikern wie Pétion und Robespierre. "Plus un homme est faible et malhcureux «, meinte Robespierrc, „plus il a de besoins, plus les prières lui sont nécessaires. «s Wenn jedoch die Schwachen wirklich geschützt werden sollten, dann müßte es in ihrem Interesse ein kollektives Petitionsrecht geben. Die Kritik blicb freilich ohne Folgen. Allc Versuchc, dic Entscheidung wenigstens zu vertagen, scheiterten. A Dic Mehrheit der Abgeordncten teilte Le Clapeliers Meinung: Jede Konzession an ein kollektives Petitionsrecht kam aus ihrer Sicht einem Rückfall in unannehmbare korporative Strukturen gleich.

Was dann, am 14. Juni 179r, geschah, war nur folgerichtig. Le Chapelier ergriff im Namen des Vcrfassungsausschusses das Wort's und begann seine Ausführungen mit einem Hinweis auf die sich häufenden Verstöße gegen das sich aus den Grundsätzen der Verfassung ergebende Korporacionsverbor. Vor allem die Handwerkerversammlungen hätten sich zunehmend zu fesien, mitcinander verbundenen und ganz Frankreich einbeziehenden Organisationen entwickelt. Sie verfolgten offensichtlich das Ziel, die einzelnen Arbeirer daran zu hindern, ihre Arbeitsbedingungen selbst auszumachen, versuchten also, die Arbeiter zu zwingen, sich an die in den jeweiligen Versammlungen beschlossenen, in Verhandlungen mis den Meistern verbind́lich festzuschreibenden Beschäfrigungsbedingungen zu halten. Zwar müsse es allen Bürgern crlaubr sein, sich zu versammeln. Dennoch ginge es nicht an, Versammlungen für zulässig anzusehen, die nur aus Bürgern bescünden, die einem bestimmten Beruf angehörten und im Namen angeblich gemeinsamer Interessen einberufen würden.

Daran schließen sich jene berühme gewordenen, eingangs zitierten Bemerkungen, die an das Ende aller Korporationen ebenso crinnern wie an den unbedingeen Vorrang der individuellen Intercssen. Le Chapelier verläßt damit den Kontext der konkret von ihm angesprochenen Vorfälle und begibt sich auf die Ebene der von der Nationaiversammlung immer wieder in den Mittelounkt aller Debatten gesteliten konstitutiven Grundsätze einer individualistischen Gesellschaft: Sic kann nur auf der Grundlage ciner Regelung funkrionicren, die dirckı an die individuellen Erwartungen knüpft, sie aufnimmt und in vom cinzelnen akzcpticrtc, verbindliche Verhaltensregeln umsetzt. Deshalb erklärt Le Chapelier die zwischen den jeweils konkret betroffenen einzelnen frej ausgehandelte Vereinbarung zum einzig legitimen Regelungsinstrument und aus dem gleichen Grund sicht er in der Unterstützung kranker und hilfsbedürfriger einzelner eine primär der Nation obliegende Aufgabe. ${ }^{46}$ Der

4) Archives Parlementares, a.a. O., 68s

44 A rchives Parlementaures, a. a. O.,685

4) Archives Parlementaires, lère séric, 27 (1886) 210.

46 Archives Parlementerics, 3.2. O. 
cinzelne soll weder bei der Entscheidung über die Verwertung seiner Fähigkeiten noch bei der Inanspruclinahme lebensnowwendiger Hilfe in Abhängigkeiten verstrickt werden, die nur den forrschrcirenden Verlust seiner Einflußmöglichkeiten zur Folge haben könnten. Mehr denn je erscheinen daher intermediäre Gewalten als das Gegensrück zu der von der Nationalversammlung geforderten und von der Erklärung der Menschen- und Bürgerrechte sanktionierten Unmittelbarkeit der Meinungsbildung. Nichrs anderes besagr der Sacz, daß es nur noch die Inzeressen des einzelnen und die der Allgemeinheit gebe. Nur der gegen die Gefalir einer Verfälschung der eigenen Wahrnehmung sozialer und ökonomischer Vorgänge durch den Einfluß partikulärer, korporativ vermittelter Interessen gefeite cinzelne kann seine Meinung in den gescllschafclichen und politischen Diskursen einbringen und so das Allgemeininteresse mitdelinicren.

Le Chapelier gibt sich deshalb niche mit einer abstrakten Kritik zufrieden. Er verlangı gezielte Gegenmaßnahmen und legt entsprechende Vorschläge vor. Sie bestätigen das Korporationsverbor und sichern es zugleich durch eine Reilie von Sanktionen ab. So werden zunächst alle organisatorischen Vorkchrungen untersagt, die Versammlungen der Angehörigen eines bestimmen Berufes gleichsam perpecuieren und damit in einen Verband überführen. Mit der gleichen Rigorosität werden dann alle Absprachen für nichrig erklärt, die ein gemeinsames Vorgehen bei der Verwertung der Produktionsmitcel oder der Arbeitskraft vorsehen. Ob eine einheitliche Regelung durchgesetzt oder dic Verwertung kollekriv verweigen werden soll, spielt insofern keine Rolle. Beides ist gleichermaßen unzulässig. Beschäfrigungsverbote für alle, die sich an den untersagten Berufsvercinigungen beteiligen sowie Geldbußen und Gctängnisstrafen für jeden, der erwa durch Drohungen die individuelle Entscheidungstreiheir gefährdet, sollten schließlich dem Korporationsverbor den nörigen Nachdruck verleihen. Le Chapelier sprach sich damit offen auch und gerade für cinc Kriminalisierung sämtlicher Bestrebungen aus, die Verrrags- und Gewerbefreiheit mit Hilfe von Berufsvereinigungen zu unterlaufen. Wer mit koilektiven Maßnahmen in den individuellen Entscheidungsprozeß eingreift, verstößt eben nicht mehr "nur" gegen die elementarsten Verfassungsprinzipien, sondern muß als "perturbateur du repos public" verfolgt und bestraft werden. Ebenso folgerichtig erscheint es dann, alle die Ausübung der Gewerbefreihcir behindernden Ansammlungen von Arbeitern als satcroupements fédicieux« zu disqualifizieren, kurzum als kriminellen Aufruhr auszugeben.

Diskutiert wurde kaum. Die wenigen Abgeordneten, die sich zu Wort meldeten, hatten weder gegen die Ziele des Entrvurls noch gegen dessen Bestimmungen erwas einzuwenden. Yhnen ging es vor allem darum, einzelne Formulierungen auf mögliche Widersprüche mit anderen Regelungen, besonders dem Dekret über die Versammlungsfreiheir zu überprüfen und die ihrer Meinung nach zu restriktiven Bessimmungen über den Anwendungsbereich des Geseizes weiter zu fassen. Le Chapelier erklärte darauhhin, die vorgeschlagenen Bestimmungen wären auf alle Berufsverbände anwendbar, auf den konkrer ausgeübten Beruf käme es deshalb nicht weiter an. ${ }^{47}$ Die Abstimmung fand noch am gleichen Tag statt. Der Entwurf wurde unverändert übernommen, und zwar einstimmig. Etwas über zweiundzwanzig Monate nach jener, von Le Chapelier geleiteten Sizzung, in der die Narionalversammlung zum ersten Mal dic Unvereinbarkeit intermediärcr Gewaiten nit einer der Souveränität des cinzelnen verpflichteten Gesellschaft proklamierte, bekräftigte

47 Archuves Parlementares, a. a. O., 211. Die Nationalvcrsammlung hal es allcrdings kure darauf, am 20. Juli 1791, fur norwendig gehalten, cine ànliche Regclung lür den Bcresch der Landowirischafr zu treffen. Archives Parlemeneares. lërc séric, $28(1887) 449$ lf., 456 l. 
sic mit diesem auf Antrag Le Chapeliers zustandegekommenen Geseť dic absolute Prioritit individueller Entscheidungen genauso wie die strikte Wcigerung, intermediäre Interessen anzuerkennen und in rvelcher Form auch immer hinzunehmen.

3.

Die lange Vorgeschichec läße deutlich erkennen: die Loi le Chapelier war keines jener Gesetze, die gleichsam aus der Stimmung des Augenblicks entstehen und deshalb bescenfalls den Anspruch erheben können, momentane, in ihrer Bedeutung daher höchst beschränkte Reaktionen zu illustrieren. ${ }^{48}$ Daran ändern auch die im Bericht Le Chapeliers ebenso wic in der anschließenden Diskussion vorkommenden Hinweise auf den zunehmendicn Widerstand, vor allem der Arbeiter, gcgen das Korporationsverbot nichts. Schaut man genauer hin, dann xeigt sich selir bald: Von einer auch nur im encferntesten alarmierenden Situation konnce zumindest im ersten Halbjahr 1791 keine Rede sein. ${ }^{49}$ Die ökonomische Lage war noch relativ stabil. Größere Protestaktionen sind allein schon deshalb nicht «u verzeichnen. Zudem, wo es tatsächlich zu nennenswerten Arbeitsniederlegungen kam, hatcen sie nichts mit der Korporationsdiskussion zu tun. Sie hingen viclmchr mit der Erwarsung der Arbeiter zusammen, nun endlich auch von der günstigen wirtschafulichen Entricklung zu profitieren und nicht ständig auf die im Februar 1791 von der Nacionalversammlung beschlossene Senkung der Verbrauchsstcucrn verwiescn zu werden.

Gewiß, die von Le Chapelier in seinem Bericht zitierte Pariser Stadtverwaltung hatte in ihrem an die Nationalversammlung gerichteten und in der Forderung nach einer das Korporationsverbot konkretisicrenden gesetzlichen Regelung gipfelnden Bericht mir Hinweisen auf gewaltsame Auseinandersetzungen nicht gerade gespart, präzise Angaben aber vermieden, "Das Schreiben liest sich aber weit eher wic eine jener zeitlosen Ansammlungen rhetorischer Sentenzen, dic zumeist mit Bemerkungen über die immer weiler um sich greifende Gewalttätigkeit beginnen, mit Hinweisen auf die zunchmende Angst der Bevölkerung und die Flucht wohlhabender Bürger in andere rulyigere Gegenden fortfahren, um dann der ernsten Sorge über dic weiterc Entwicklung Ausdruck zu geben und mit einem Appell an den Geseczgeber zu schlicßen, möglichse sofort zu intervenieren. Die Eingabe der Meister ${ }^{51}$ bietet cbenfalls keinc Anhaltspunkte für sich ständig verschärfende Konflikte. Mehr als die Feststellung, sie, die Meister, seien, genauso wie alle übrigen Bürger, das Opfer einer Aufruhr der Arbeiter, die ihre einseitigen Vorstellungen und Interessen durchzusetzen suchten, enthäl die Eingabe nicht.

Aber auch die Ende Mai i 791 , nur wenige Tage also vor dem Beginn der Debaute über den Antrag von Le Chapclier, eingereichte Perition der Gesellen ${ }^{\prime 2}$ läßt nicht auf weitreichende Auseinandersetzungen schließen, oder gar auf den Willen, den Konflikt zu eskalieren. Sie offenbart im Gegenteil eine siefe Unsicherheir, ja Ratlosigkeit. Die Gesellen erinncm zunächst an dic von der Nationałversammiung verkündete Abschaffung aller Privilegien, fragen dann, ihnlich wie die 340 Arbeiter von Sre. Geneviève in ihrem an Marat gerichteren, am r2. Juni $179 \mathrm{r}$ im Ami du

$48 \mathrm{Vgl}$. freilich Martin. Les associations ouvricres au XVIie siccle (5700-1792) (1900) 220 ff. und demgegenüber Soreau, Annales hissonques de la Rivolution françalse 8 (1931) jooff., Bouvicr-Ayam, Histoure, 3.2. O., 705 .

19 Dazu insb. Soresu, Annales histonques de la Révolucion françase 8 (1931) 301: Dollèans/Dehove. Historre 133 .

so Dazu Buclice/Roux, Historre parlementaure de la Révolucion françuse. Joumal des Assemblèes Natsona les depus 1789 jusqu'en 1815 10 (1834) 106

51 Abgedrucke bei Martun, Associations 232 f.

\$2 Abgedruckt bei Jaures. Historre socialiste de la Révoluton fraşasse I (1901) 619 f. 
Peuple publizierten Brief, nach dem Nutzen, den diese Entscheidung für sie gehabr hat und lassen sich schließlich auf eine überaus juristisch anmutende Argumentation ein: Sic geben zwar einerseics zu erkennen, daß sic durchaus für das Korporationsverbor sind, meinen aber andererscits, die von ihnen gcbildere Koalition könne gar nicht darunter fallen. Ihre Zusammenarbeit beruhe auf der freien Entscheidung aller Beteiligten, trage mit anderen Worten genau dem Prinzip Rechnung, dem die Nationalversammlung den Vorrang cingeräumt habe und verfolgc im übrigen nur das Ziel einer gcgenseirigen Hilfelcistung.

Kurzum, Belege, dic es rechtfertigen könnten, die Loi lc Chapelicr als eine schnell improvisierte, eigens auf die Bekämpfung sich häufender, von den Arbeitern ausgelöster Unruhen abgestellre Maßnahme auszugeben, finden sich, allen gegenteiligen Behauptungen zum Trotz, nicht. Für die Nationalversammlung stand eben nicht die Reakion auf wie immer bewertete Einzelcreignisse auf $\mathrm{dcm}$ Spiel. Den Abgeordneten ging es vielmehr zuvörderst darum, die für sie einzig akzeptablen Gestaltungsprinzipien gesellschaftlicher Ordnung einmal mehr klarzustellen und zugleich jeden Zweifcl am Geltungsanspruch dieser Grundsäzze auszuschlicßen.

Gcnau dicser Wunsch drückt sich auch im Abstimmungsergebnis aus. So sehr die Meinungen auscinandergingen, ja aufeinander prallten, sobald Einzelaspekte der politischen und ökonomischen Entwicklung diskutierı wurden, so schnell war der Konsens wiederhergestellr, sobald die Grundbedingungen einer individualistischen Gesellschafr zur Debatre standen. Das Schweigen Robespierres überraschr deshalb genausowenig wie die einstimmige Verabschiedung des Geseczes." In Gegenceil, Verwunderung wäre nur bei einem anderen Diskussions- und Abscimmungsverlauf angebracht.

Politisch hacte Rousseau das Argumentationsfeld bereits abgesteckt." Eine einwandfreie, den Willen aller einzelnen wirklich widerspiegelnde "voloncé générale“ und die Tolerierung intermediärcr Gewalten schließen sich in scinen Augen gegenseitig aus. Solange intermediäre Gruppierungen hingenommen würden, könnte weder der cinzelne scinc Meinung frei und ungehindert bilden, noch hätten die Individuen eine Chance, ihren Entschcidungsprozeß gemeinsam in die nvolonté générale $\alpha$ umzusetzen.

Keiner der Abgeordneten und erst rechr nichr Robespierse har dicse Feststcllung jemals angezweifelc. Die unterschiedliche Reakuion auf den Vorstoß Le Chapeliers zum Pecitionsrecht ist kein Gegenargument. Robespierre" und Marat ${ }^{56}$ mögen zwar vehement widersprochen haben. Sie wollten aber mit ihrer Kritik nicht den Konsens über die Unvereinbarkeit intermediärer Gewalten mit der auch von ihnen akzeppierten und energisch angestrebten individualistischen Gesellschaft aufkündigen. Beiden ging es vielmchr darum, in Kenntnis dicser Übereinscimmung die Bcdingungen anzusprechen, unter denen es wirklich gelingen könnte, die individuelle Meinungsbildung sicherzustellen und damit zugleich der Souveränität der Individuen uneingeschränkt zur Geltung zu verhelfen. Beider Intervention war daher kcin abstraktes Plädoyer zugunsten eines allen Vereinigungen unterschiedslos zuzugestehenden kollektiven Petitionsrechts, sondern eine gezielt auf die politischen Assoziationen zugernünzte Forderung. Dahinter stand die besonders von Maras" hervorgehobene edukative Funkrion der politischen Clubs, genauer, die ilnen zugedachre Aufgabe,

53 Vgl. Frcilich Jaurés, Hiscorre socialiste a a a. O., Siof.

54 Du contras social ou Pnncipes du droit poliuque (Ausgabe Garner 1960) 243 f., 246. 250.

ss Archives Parlementaires, lère série, 2s (1886) 684,69r, 692.

s6 Ami du Pcuple vom 12. Ma1 1791.

7 Ami du Pcuple vom 4. Márz 1791. 
jedem ihrer Mitglieder die Chance zu geben, sich über die Teilnalimc an den internen Debatten selbst eine dann auch öffentlich zu vertretende Meinung zu bilden. Dic politischen Vereinigungen wurden, anders ausgedrückr, von beiden als Brücke verscanden, die den Übergang aus einer autoritären in eine vom freien Diskurs aller einzclnen geprägten Gesellschaft ermöglichen sollıc. Eben diesc ebenso spezifische wic einmalige Funktion der politischen Vereinigungen rechtertigte es in den Augen beider, sic niche wic die übrigen Assoziationen zu behandeln, ihnen also doch ein auf der von ihren Mitgliedern gestalceten, mithin deren Ansicheen ausdrückenden Diskussion beruhendes Pecitionsrechr zuzubilligen.

Ökonomisch hatce Turgot den A rgumentationsverlauf vorgezcichnec. In der Präambel zum Edikt von 1774 waren die Gründe für eine uneingeschränkte Vertrags- und Gewerbefreiheic formulicrt, im Edikt vom Januar 1776 die wichtigste Konsequenz gezogen worden: die Abschaffung aller Zünfre. ${ }^{8}$ Genaugenommen nahm daher die Nationalversammlung die Debatre dort wieder auf, wo sie durch die Abdankung Turgots und dic Rückkehr Neckers untcrbrochen worden war." Kein Wunder, wcnn deshalb d'Allarde in den einleirenden Bemerkungen zu scinen Geserzesvorschlägen vom is. Februar s791 ausdrücklich an den "Minisrer-Philosophen a erinnert und ebenso explizit dessen Überlegungen aufgreifc. ${ }^{60}$ In Wirklichkeit bekräfrigt er damit keincswegs nur die ökonomischen Maximen der Physiokraten. Seine Ausführungen lesen sich vielmehr über weite Strecken wie cine Wiedergabe der Reflexionon Adam Smiths. ${ }^{61}$ Gemeinsam ist allen die Überzcugung, daß cs zuvörderst, um d'Allardes Formulierungen aufuugreifen, darauf ankomme, dic wiberté générale "an die Srellc einer "multitude de perirs privilèges exclusif́s qui se croisent en multiplient sans cesse les conecstations et les procès, et qui entrainent une stagnarion considérable des capitaux ${ }^{6 x}$, abcr auch jener Vielzahl von „droits destructeurs de toure industrie, aussi inconséquents que ryranniquesa zu setzen. Nur dann, so meinen sie, könne das Eigentum seine Doppelfunktion voll entfalten, einerseits die bestmögliche Verwirklichung der individuellen Intcressen garantieren und andererseics dic ökonomische Prosperität sowie die moralische Stabilität der Gesellschaft sicherstellen. Der citoyen-propriéraire ist, wic die Nacionalversammlung nur wenig später, in der Debatte vom I t. August $179 \mathrm{I}^{63}$, emeur bestätigen sollte, weit mehr als nur Motor der ökonomischen Entwicklung. Sein Eigentum ist in Wirklichkeir das Fundament jener Kombination von stets präsentcm persönlichem Interesse und konstant gervährleisterer Unabhängigkeit, die erst die Fähigkeit vennitcelt, nicht nur die eigenen Belange, sondern auch und gerade das Allgemeininteresse korrekt beurreilen zu können.

Was sich bei Turgot schon andeuter, steht für Adam Smith genauso wie für d'Allarde außer Zweifel: Vom Eigenturn kann und darf keineswegs nur dann die Rede sein, wenn etwa der einzelne über Grundeigentum verfügt. "The property which every man has in his own labour a ist viclmehr in den Worten Adam Smiths wthe original foundacion of all ocher Property «. ${ }^{\sigma_{4}}$ Ähnlich drückt es d'Allarde aus:

¿8 Euvres, liesg. von E. Daurc und H. Dussard, $2(1844) 302$ ff.

ig So gesehen, lißis steh in der Tat feststellen, daß die Revolusson noch vor ihrem Beginn sehon xu dreı Vierteln vollzogen war. Furet, Penser la Révolution françalse $(1983)$ 185; vgl, auch Tocqueville, Euvres complètes II: L'Áncien Régime 1 (1952) 168 ff., i is ff.

60 Anchives parlementaires, lèré séric, $23(1886) 199$.

61 The Weahh of Narions i (Ausgabe J. M. Denr \& Sons, Neudruck 1960) 107ff

62 Archives Parlementaircs, a.2. O., 201. ( $*$ Vielz.2hl cxklusiver klancr Vorreches, dic sich uberschneiden und unentwegt Stratıgkeiten und Prozesse verviclfachen sovve zu ener betrachelichen Stagnation der Kapıalien (ühren. $x$ ).

63 Archives Parlementaures, lère sénc. 29 (1888) j50ll.

64 Wealth of Nations a. a. O., I10. 
- La faculté de travailler est un des premiers droits de l'homme. Ce droit est sa propriété ......65 Für Adam Smich ${ }^{66}$ ist daher genauso wie für d'Allarde ${ }^{67}$ dic Arbeitskraft "the most sacred and invoilablca Eigentumsform, nla première propriććé la plus sacrée, la plus imprescriprible*. In dem Augenblick aber, in dem die Arbeitskraft jedem anderen Eigentumsgegenstand gleichgestellt wird, veränderı sich auch die Marktstruktur. Die Uncerschiede in der Eigentumslorm können sich auf die Zutrittsbedingungen nicht mehr auswirken. ${ }^{68}$ Wo anderc Vermögensobjektc nichr varhanden sind, muß es genügen, dic Arbeitskraft einzubringen: $x$ The patrimony of a poor man lies in the strength and dexterity of his hands ....

D'Aliarde wußre jedoch ebensogut wic die übrigen Abgeordneten, daß der zum Eigentümer erklärte Arbeiter nicht ohne weiteres aú eine Stufe mit allen anderen Eigentümern gestellt werden konnte. Die Verfügung über dic Arbeitskraft mag die Grundform allen Eigentums sein, sie vcrleiht trozzdem dem Arbeiter nicht das Gewicht und den Einfluß, den die übrigen, nicht ausschließlich auf die Verwertung ihrer Arbeitskraft angewiesenen Markcteilnehmer für sich in Anspruch nehmen können. Schon zu Turgors Zeiten hatte etrva Condorcet jeden Verdacht scharf zurückgewiesen, die Konsequenzen der unterschiedlichen materielien Lage würden verniedlicht oder gar verkannt. "Vous dites", schricb er an die Adresse der Kritiker Turgots, "que nous sommes tentés de regarder les riches comme des êtres d'une nature différentc, que leur grandeur est une magie qui nous en impose. .. Nous sentons que si leur argent leur donne la facilitć d'acheter des jouissances dont nous sommes privés, il nc leur donne aucun droir d'obtenir sur nous des distinctions ou des préférences .... $\otimes^{70}$.

Weder Condorcet noch d'Allarde sahen freilich das Korrektiv in staatlichen, auf den Ausgleich der Eigentumsunterschiede abzielenden Eingriffen. Nicht umsonst nennt die Erklärung der Menschen- und Bürgerrechte vom 26. August 1789 das Eigentum an zweiter Stelic, gleich nach der Freiheir, bei der Aufzählung der wnatürlichen \& und "unveräußerlichen Menschenrechte. Die Emanzipation des einzelnen isc eben nicht eine Befreiung vom Eigentum, sondern im Gegenteil nur als Befreiung des Eigentums denkbar und realisierbar. Der Ausgleich läßt sich unter diesen Umständen lediglich auf dem Weg über die Aufhebung sämllicher, die politische und ökonomische Berätigung des einzelnen einschränkenden legislativen und administrativen Hindernisse schaffen. Wo alle Bürger über die gleichen Rechte verfügen, ist die von den Bürgern gemeinsam formulierte "volonté générale" das jederzeit aktivierbare politische Gegengewicht gegen jeden Versuch, politische Privilegien und damit einseitige Herrschaft zu restiruieren. Wo zudem die freie Verwertung der Arbeitskraft den Arbeiter zum citoyen-proprićraire, ja mehr noch, zum citoyen-actionnaire, um einen Ausdruck von Sieyčs zu benutzen"1, macht, erscheint die konsequente Verfolgung der eigenen Interessen am Markt, gestürz.t und abgesicherr durch die politische Souveränicär, als das einzig akzeptable Mittcl, um über eine sich

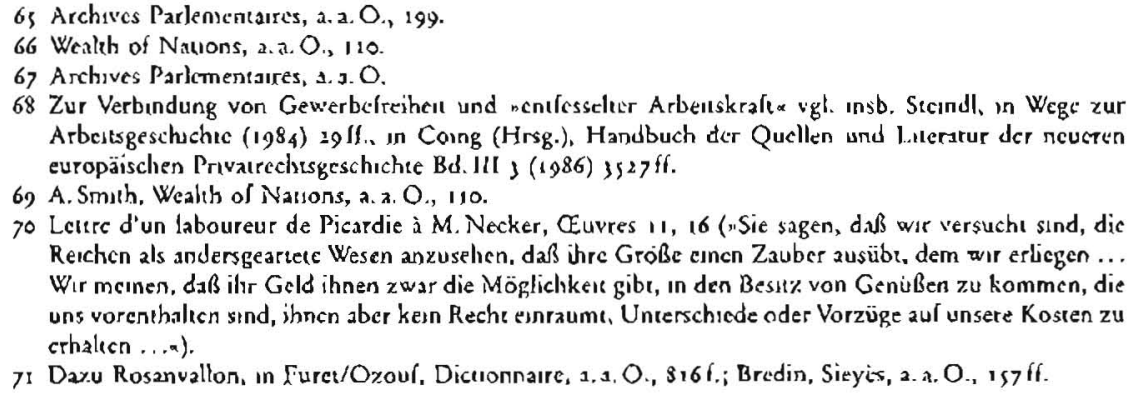


ständig verbessernde materielle Situation die Diskrepanz der Vermögensverhälonisse fortschreitend zu beheben.

Für d'Allarde stand aber ebenso wie für die übrigen Abgeordneten fest: Dic Verwerung der Arbeitskraft kann die ihr zugedachte Funktion nur erfüllen, wenn der einzelne selbst die Bedingungen zu bescimmen vermag, unter denen sie eingesetzt werden soll. Dic Vertragsfreiheit ist daher das gleichsam "natürliche Korrclat der Eigentumsfreiheic. Erst über die Vertragsfreiheit gewinnt der einzelne jenes Maß an Selbständigkeit, dessen er bedart, um jedes Eigentum, also auch und gerade die Arbcitskraft, in eincr seinen Interessen entsprechenden Weise nutzen zu können. Eben deshalb beläßr es beispielsweisc die Verfassung vom 24. Juni 1793 nicht bei der Bekräftigung des Eigentumsrechis, sondern hebr genauso das Rechr jedes einzeincn hervor, über scine Arbeitskraft zu verfügen, macht aber auch deutlich: "La loi ne reconnait point de domesticité; il ne peut exister qu'un engagement des soins et de reconnaissance entre l'homme qui travaille et celui qui l'emploie. " ${ }^{22}$ Der oksroyicrte Status weicht so der frei vereinbarten kontraktuellen Bezichung. Konsequenterweise wird sich deshalb einige Jahre später der Code civil gar nichr lange beim Arbeitsverhältnis aufhalten. Weil cs nur eines unter vielen Vertragsverhältnissen ist, bleibt es bei dem auch ansonsten geltenden Grundsatz: Die Entscheidung über Abschluß, Inhalt und Aufösung liegr bei den Beteiligten. Der Gesetzgeber kann, ja darf nicht mehr tun, als dies zu unterstrcichen."3

Die Encscheidung für einen dem citoyen-propriétaire völlig überlassenen und von seinen frei gestalteten Verträgen beherrschten Markt schließt dic Absage an jeden Vcrsuch ein, wcm auch immer das Recht einzuräumen, stevemd in das Verhalten des einzelnen einzugreifen. Weder geht es an, ihm vorzuschreiben, wann er gleichsam den Markt betreten darf, noch kann es gestactet sein, die individuelle Aktivität in den Rahmen einer verbindlichen Überwachung zu zwängen. Mic der Vorherrschaft einer sich in der Verragsfreiheit ständig von neuem aktualisierenden Eigenrumsfreiheit endet daher die Regelungsbefugnis der Korporationen cbenso wie der Geltungsanspruch der zahlreichen dirigistischen Vorgaben. "... la surveillance de la loi doit commencer là où cesse celle du citoyen a stellte deshalb d'Allarde fest ${ }^{74}$ und fügte hinzu, nichts garantiere eine bcssere Überwachung als die Konkurrenz, sie allein sélève, perfecrionne las talents, qu'unc police despocique décourage et héerit.

Wenn mithin d'Allarde in einer am 17. September 1791 vor der Nationalversammlung gehaltenen Rede erklärte: $\rtimes . .$. ne doutez pas que les principcs que vous avez posés, et qui se propageront comme la lumière, n'éclairent tous les peuples sur leurs

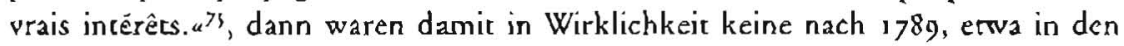
politischen Clubs oder der Nationalversammlung entwickelten Prinzipien gemeint, sondern die bercits von Turgor formulierten Grundsärze. Der eigenrlich entscheidende Beitrag der Nationalversammlung lag anderswo: in der konsequenten Ver-

72 Art. 1 Nr. 18 ("Das Gesetz kenni ken Gesinde; zwische'n demjenigen, der arbeitel und demjenigen, der ihn beschäftugt, kann es nut eıne Verpflicheung geben, Dienste zu crbnngen und diese zu crelohnin.") 73 Dazu Tissier, in Le Codc civil - Livre du cemeenaure l (1904) 7Ifl; Símus in Zacher/Simuts/Kübler' HopeTeubner, Verrechtlichung von Wirechaft. Arbeıt und sozıaler Solidarıtär (1984) 74 If. Mit Reche meint deshalb Furer. La Révolution (1988) 88, in Futet/Oz.ouf Dictionnaire, a. a. O.. 132. in der Nache

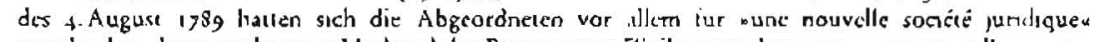
entschuden, deren wichesgses Merkmal der Begenn fener Zivilgeser/_gebung ses, osnstaurant l'universalité de la lor dans la sphère de la sociécia, so daß es eigentlich nur noch Nuancen zwischen den - discussions de la Convention et celles du Consulat sur le futur Code civila gabe.

74 Arcluves Parlementares, lère sénc, 23 (1886) 200.

75 Archıves Parlementares, lère sérıc, 30 (1888) 739 ( $\ldots$ zweifoln Sie nıcht, daß die Prinzıpıen, die sıe gesetze haben und die sich wic dis Licht verbreitton werden, alle Volker über ihre wirklichen Interessen uufklären werden ....\%). 
knüpfung des ökonomischen mit dem politischen Liberalismus. Dic Freiheit des einzelnen endete nichi bei der Gewcrbefreiheit. Das Recht, jederzeit und ungehindert die den eigenen Vorstcllungen und Interessen entsprechende wirschafuliche Tätigkeit ausüben zu können, wurde vielmehr umgekehr als Appendix der Freiheit verstanden, über die cigene Enewicklung ebenso wic über die der Gesellschaft zu bestimmen.

Genau diese Verknüpfung rechtécrtigt nicht nur d'Allardes Feststellung: „La liberté ne connait enfin plus de linites! ${ }^{76}$, sondern erklärt auch die einstimmige Billigung des Korporacionsverbores. Weil, anders ausgedrückr, die ökonomischen Maximen nicht selbstïndig präsentiert und debatriert wurden, vielmehr durchweg im Gefolge der die policische Emanzipation des Bürgers proklamierenden Prinzipien, konnce es etwa Robespicre gar nichr schwerfallen, sich jeder Bemerkung zu enthalten und den Gesetzesvorschlägen uncingeschränkt zuzustimmen. Der hoino politicus ging für ihn wie für viele andere Abgeordnete dem homo oeconomicus vor. Der ouvrier war in seinen Augen genauso wie der maître oder der commerçant vor allem anderen citoyen. So betrachtet, war eines mit Sicherheit unzulässig: die Restrikcion der Reflexion auf den ökonomischen Bereich. Auf die wirtschaftlichen Entscheidungsprozesse beschränkte Korrekturen hätren in den Augen Robespierres die marerielle Lage dor Arbeiter möglicherweise durchaus verbessert, zugleich jedoch parrikulärcn Interessen nur noch mehr Aufrricb verliehen und so dazu beigetragen, die politischen Unterschiede weit eher zu perpetuieren als sic aufzuheben. Deshalb kam es ihm in erster Linie darauf an, die ökonomischen Veränderungen in den Kontext der politischen Modifikationen zu stellen, also die politischen Rcchte der Bürger zur unverzichtbaren Grundlage aller Überlcgungen übcr dcren ökonomische Rechtc zu erkłären. Solange diese Reihenfolge respektiert wurde, gab es auch keinen Anlaß für Kontroversen. Im Gegenteil, die zunächst von d'Aliarde und dann von Le Chapelier aufgegriffenen und zu Geseczesmaximen erhobenen Prinzipien waren für Robespierre cbenfalls die natürliche Ergänzung der prioritären policischen Grundsätze."7 Das Korporationsverbot beugte der Entstehung sozialer Strukturen vor, dic der gerade für den politischen Bereich unerläßlichen Autonomie des Bürgers zuwiderlaufen; die Gewerbefreiheit sicherte und konsolidierte die Entfaltung des Eigenrums, stützte aber damit eine der zentralen Voraussetzungen für ebenso selbständige wie vernünfrige ökonomische und politische Encscheidungen des Bürgers ab.

Bleibc der Widerspruch Marats; für nahezu jeden, der in der Loi le Chapelier nur den Verrat an den Revolutionsprinzipien sieht, der wohl eindrucksvollste Bewcis für die Richrigkeir dieser Thcse. Kein Zweifel, Marat hatce schon aus seiner Ablehnung der Vorschläge d'Allardes keinen Hehl gemacht. ${ }^{78}$ Seine Argumence passen freilich kaum ins Konzept derjenigen, dic sich auf ihn berufen. Um mic jenem immer wieder ziticreen Artikel vom 12. Juni 1791 anzufangen: Marat registriert zunächst den Protest der Maurer von Ste. Geneviève, beschränkt sich dann allerdings auf einige kurze Bemerkungen, die ebenso scharf wie die Arbeiter selbst, die von dicsen monierte, durch einscitige Bereicherung und wachscnde ökonomische Disparität gekennzeichnete wirtschaftliche Entwicklung vcrurteilen. ${ }^{79}$ Von einer Auseinandersetzung mit der geplanten geseczlichen Regelung kann dagegen keine

76 Archives Pariemencaures, A. a. O.

77 Vgl, auch Waltcr, Maxumilian de Robespierre (Neuauflage 1989) insb. I2Iff. In der Forderung nach esnem unbedingten Vorrang der polituschen Enischeidungen drücke sich auch und geradc die Überzeugang aus, daß eine von der Vernunft geleitete und auf die Firziehung des Bürgers bedachec Gescllschal nur aul dicse Weise ihr Zicl, die Freihele aller, warklich crreachen kann; vgl. auch Elisaberth und Rober Badinter, Condarece (1988) 252

78 L'Amı du Pruple vom 16. Mai 1791.

79 L'amı du Peuples. 
Rede sein. Etwas anders sieht der Kommentar im "Ami du Peuplea vom 18. Juni

1791 aus. Marat spricht in der Tar das Korporationsverbot an. Schon die ersten Bemerkungen zeigen freilich, daß es ihm in Wirklichkeit um erwas ganz anderes geht. Was scine Kritik liervorruft, ist niche das Verbor selbst, sondern die Absicht, die er dahinter vermuter: das Recht der Bürger einzuschränken, ihre Meinung äußern zu können. Er belürchtet ebenso wie bei der Ablchnung des kollckriven Petitionsrechts eine weicere Reduktion dor Möglichkeiten einer gemeinsamen Mcinungsbildung und damir auch der Chance, gemeinsam auf die Nationalversammlung Einfluß nehmen zu können. Den Abgeordneten wirft er daher vor, nur eines wirklich gewollt zu haben: wisoler les citoyens, et les empêcher de s'occuper en commun de la chose publique. ${ }^{80} \mathrm{Kurzum}$, das Korporationsverbor ist keineswegs der Gegenstand, sondern lediglich der Anlaß seines Protests. Für Marat sind die Korporationen nur der Vorwand, um die politischen Vereinigungen zu disziplinieren, noch genauer: sie ihrer Doppelfunkrion zu berauben, einerseits dem im Emanzipationsprozeß befindlichen Bürger gegenüber einc cdukative Aufgabe wahrzunehmen und andercrseits den Entscheidungsablaul in der Nacionalversammlung kontrollierend zu begleiten ${ }^{8}$. Auch der zweite, ebenfalls im Mai 1791 publizierte Arrikel gibc also letztlich nichts für die Bchauptung her, Marat habe sich anders als die Nationalversammiung und in offenem Gegensatz zu Loi le Chapelier für Arbeiterkoalitionen ausgesprochen.

Was er von ilnen, ja von Benufsvercinigungen übcrhaupt wirklich hielt, läßs sich dagegen durchaus seinen früheren, vor allem im Zusammenhang mit den Vorschlägen d'Allardes gemachten Äußerungen encnehmen ${ }^{82}$. Sie geben zunächst so viel zu erkennen: Marat war ein genauso entschiedener Gegner aller intermediären Gruppen wie ecwa le Chapelier und d'Allarde oder Robespiere, Pétion und Courhon, und zwar aus just den Gründen, die auch die Nationalversammlung veranlaß̧ hatten, eine eindeutig ablehnende Position einzunelamen. Die Entscheidungsfreiheit des einzelnen sorvic scin Rechr, dic politische und soziale Entwicklung zu bestimmen, sollte unter keinen Umständen gefährdec werden. Anders aber als d'Allarde odcr Robespicrrc war Marat bereit, im ökonomischen Bcreich dirigistische, dic Souveränität des einzelnen unstreitig einschränkende Eingtiffe hinzunehmen. Sein Hauptargument: Mit der Forderung nach Gewerbefreiheit verzichreten die Abgeordneren auf jeglichen Kompetenznachweis. Die Folge: "Tous les ouvrages de l'ar doivent promptement dégenćrer en savetage ... Chacun pourra s'établir pour son comprc sans être asujetui à faire preuve de capacité. $\alpha^{83}$ Ja, noch schlimmcr: "À pcine un apprenti saura-t-il croquer quelque ouvrage, qu'il cherchera à faire valoir son industrie et qu'il ne songera plus qu'à s'établir ou à valeter pour trouver des pratiques ou des chalands", "tout crafic dégénère en fripponericu. ${ }^{\delta_{4}} \mathrm{Sow}_{\mathrm{O}} \mathrm{Sh}$ im Interesse der Gesellschaft als auch und gerade um dor Arbciter willen müsse es deshalb verbindliche, den Sachverscand garantierende sowie auf die einzelnen Berufc zugeschnittene Zugangs- und Ausübungsbedingungen geben. Ohnc eine solche

80 L'Nmi du Pcuple 8.

81 Daß Marat durchaus Grund au solchen Befürcheungen hates, zeigi ssch niche suletzt an manchen. sich

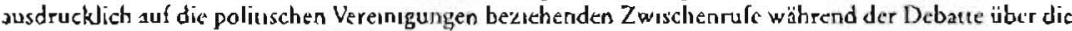
Lat le Chapelicr. Archives Parlementares, lère xćric, 27 (1887) 212.

\$2 L'Amı du l'euple vam 16. Mai 1791.

s] "Samtliche Handwerkserceugnisse müssen alsbald yu Plusch degenincren ... Ieder wird auf eigene

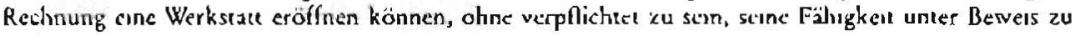
siellen.*

\$4 -Kaum, d 3 can Lchrling ivciß, wic man en Werk entwirf, wird cr versuchen, acin Gewarbe zu verweren und nur noch daran denken, sich niederzulassen oder krsecherisch um Aufurige und Kunden zu werben, jedcr Handel wird zuin Betrug." 
Regelung könne es weder gclingen, den Kansumenten wirksam zu schützen, noch dem Arbeiter jenes Mindestmaß an Wissen zu vermitteln, das ihn erst dazu befähige, seine Belange, in den von ihm abgeschlossenen Verträgen wirklich wahrzunehmen. Die Abgcordneten hätten daher, stat alles in Frage zu stellen, den Rat »des hommes instruits sur les choses* einholen müsscn, "pour s'attacher à corriger uniquement les abus«. Für Marat ist es insofern niche "a manifest encroachment upon the just liberty both of the workm an and of those who might be disposed to emplay him " 85 , vorzuschreiben, unter welchen Umständen, wer welcher Tätigkeit nachgehen darf. Erst recht kann aus seiner Sicht nicht davon die Redc sein, daß: „The affected anxiety of the law-giver lest they should employ an improper person is evidently as impertinent as it is oppressive. ${ }^{86} \mathrm{Im}$ Gegenteil, ein Gesetzgeber, der dies nicht tur, verfehlt in den Augen Marais eine sciner wichcigsien Aufgaben.

Deuclicher läßr sich dic Meinungsdivergenz kaum ausdrücken: Während die Nationalversammiung zu Turgor zurückkehrt, bleibr Marat bei Necker srehen. Policischer und ökonomischer Liberalismus fallen so auseinander. ${ }^{8}$. Die Souveränität des einzelnen wird jenseits des politischen Bereichs weitgehend den Einschränkungen unterworfen, die schon im ansonsten strikt abgelchnten absolucistisch-feudalen System gaiten. Einmal mehr crweist sich aber: Marat verteidigt keineswegs das Rechr der Arbeiter, frei zu koalieren, um ebenso frei die Bedingungen zu bestimmen, unter denen sic bercit sind, ihre Arbcitskrafe zur Verfügung zu stellen. Was Marat möchre, ist, Verfalıren sicherzustellen, die eine Tcilnahme am Produktionsprozeß von einem die Qualizär der Produkte gervährleistenden Sachverstand abhängig machen. Nur vor diesem Hincergrund und lediglich soweit es dieses Ziel erfordert, erscheinen ihm strikı auf den ökonomischen Bereich beschränkte Berufsvereinigungen akzeprabel.

Wenn deshalb die Hinweise auf Marat überhaupt einen Wert haben, dann nur weil sie exemplarisch dafür sind, wic mic der Französischen Revolution umgegangen wird. Aus einem historisclien Ereignis, das sich in einem durchaus rekonscruierbaren Koncext abgespielt hat und sich daher nur solange richeig darstellen sowie verstehen läßt, wie man dic für diesen Kontext entscheidende Elcmente kontinuierlich bedenkt, ist längst ein Stcinbruch geworden, aus dem sich jeder gerade den Stcin holt, der scheimbar am besten zu dem von ihm gerade konstruiertcn Gebäude paßr. ${ }^{83}$ Kaum verwunderlich, wenn unter diesen Umständen das Interesse am exakten Verlauf der einzelnen Entschcidungsprozesse schwindet und sich die Revolution mehr und mehr in eine je nach Legitimationsbedarf verwendbare Ahncngalcrie verwandelt. Was Danton, Saint-Juse, Mounier, Robespierre, Mirabeau, Marat, Sicyès in welchem Zusammenliang, aus welchen Gründen und mit welchen Zielen genau gesagt baben, spielt weiter keine Rolle. Jeder von ihnen ist zum Symbal für ganz bestimmte, klar festgelegte Auffassungen erstarrt, jeder von ihnen lär sich daher ohne weiteres nabrufen a, sobald es darum geht, irgendeine dieser Auffassungen mit einem Hinwcis auf die Revolucion zu untermauern oder zu widerlegen. Dann aber fällt es in der Tat leicht, unangenehme, das eigene Revolucionsbild scörende Vorkommnisse entweder, wie bei der Loi le Chapelier, als Verrat an den Revolutionsprinzipien auszugeben, oder schlicht zu verdrängen, wie etwa im Falle

8) A. Smith, Wealch of Nasuons, 3. 2. O., 110

86 ^. Smith, Wealth of Nations 1.1. O.

87 Vgl. auch Bouvier-Ajam, Historre du travisil en France depuss la Révolueron (196́g) il if.; Ozoul, in: Furci/Ozoul, Dictionnairc, a. a. O., 280 f.

83 Vgl. such Furct. Penser la Révolution, a. a. O., insb. 23 ff., 70 ff, La gauche ex la révolution au milieu du XIX: sičclc $(1986), 1$ if. 
jenes genau einstimmig gefaßten Beschlusses vom 18. März 1793, der kurz und präzise festscellc: "La Convention nacionale décrète la peine de mor contre quiconque proposera une loi agraire ou rourc autre, subversive des propriććs territoriales, commerciales et industrielles. ${ }^{89}$

4.

Nach einmal: Vorgeschichce und Geseczestext lassen keinen Zweifel aufkommen. Die Nacionalversammlung debactierte niche auf cinem Nebenschauplatz legislariver Akrivität. Mit der Loi le Chapelier definierten die Abgeordneten erneur und nachdrücklich die Konstitutionsbedingungen einer das Ancien Régime endgülrig in die Geschichte verweisenden neuen Gesellschafr. Die Loi le Chapelier ist allerdings niche nur Dokument eines prononciert radikalen, sondern auch und gerade eines offenkundig utopischen Individualismus. Allcin schon die Geschichte der VertragsFreiheit genügt, um den tiefen Wrderspruch zwischen den Entscheidungsprämissen der Nationalversammlung und der vom «Konsensw der citoyens-propriétaires gestalteten ökonomischen und sozialen Beziehungen zu verdeutlichen. Statt dirigistische Eingriffe und korporative Regclungen ein und für allemal durch vernünfrig ausgehandelre, die involvierren Intercssen konsequent berücksichrigende und sorgfälrig aufeinander abgestimmte Abmachungen abzulösen, produzierte die Vertragsfreiheit ein immer enger geknüpfres Netz staatlicher interventionen und sich ständig weiter verfescigende korporative Strukturen. Wo der citoyen-propriétaire über nicht mehr als über die "strength and dexterity of his hands" verfügt, sich also darin auch sein Verhandlungskapital erschöpfr, sind soziale Konflikte und ihre Kehrseite, zunchmend langfristiger angelegte Versuche einer staaclichen Gegensteuerung, vorprogrammiert und bleibr den Arbeitnehmern nur die Chancc, die individuelle Verhandiungsunfähigkcit über den kollektiven Verhandlungsanspruch auszugleichen. . $^{\circ}$

Der scheinbar endlose Verrechtichungsprozeß, die expandierende woh.lfahrtsstaatliche Bürokratie, dic korporatistisch mocivierten und konzipierren "Sozialpakte» demonstricren freilich nicht nur den utopischen Charakter der von den Abgeordneten postulierten Regelungsprämissen, sie bestätigen auch, so paradox dies klingen mag, die Richtigkeit jener, allen Überlegungen der Nationalversammlung zugrundeliegenden, sich in der Loi le Chapelier ebenfalls widerspiegelnden Annahme: daß sich erst der als Subjekr verstandene und akzepticre einzeine zum Individuum entwickeln kann. Nur dic Fähigkcit, sich jewcils selbst cine Meinung zu bilden sowie nach den eigenen Vorstellungen zu handeln, verleiht dem einzeinen jene spezifische Differenz, dic seine Individualitär ausmacht. Wo deshalb diese Fähigkeir in Frage gestcllt wird, entällt auch die Möglichkcit einer Individuierung. Der Verlust der Subjekteigenschaft signalisiert, anders ausgedrückt, das Ende des Individuums.

Von einer "Wiederkchr* des Individuums"' kann daher solange nicht die Rede sein, wie im gleichen Atemzug der einzelnc als Produkt eines von der staatlichen Administration genauso wie von den privaten Unternchmen nach wic vor incensiv betrieben, auf die Typisicrung und Schematisierung des indiviauellen Verbaltons

S9 Archivgs l'arlementaures, lèrc seric, 60 (1901) 292 (n Dic Conveneion mationale dekretiert dic Todessiratc gegen jeden, der eun das landwir uschafeliche, kaufmännische, gewerbliche oder das Eigentum an Grund und Boden in Frage stellendes Gesecz vorschlagen salke.a).

go Mir Reche meine deshalb Roudil, in Collin/Dhoquors/Gouverre u. a.. Le Droul capiealiste du travail (1980) 29, we travail libéré par la Révolutuon dut ètre protégé par la Républiques, umschreibe aber darnu eine keineswegs aut Frankreich beschrankec Entwicklung, vgl. Simitis, in: Verrechelichung, a. a . O., 74 If 91 Ewald, Magazinc licucrare Hche 264 (April 1989) i6. 
bedachren "Kolonialisierungsprozesses ${ }^{92}$ beschrieben wird. Der cinzclne wird cben nicht als "acteur " wahrgenommen, sondern als Rezipient von Handlungsanweisungen, die aus ihm den woptimalen «, also den jeweils vorformulierten Verhalcensprogrammen so gut wie nur irgend möglich angepaßten Konsumenten oder Leistungscmpfänger machen." Die Neutralisierung und nicht die als Störung empfundene Individuicrung des cinzelnen ist mic anderen Worcen das Ziel. Ganz gleich deshalb, ob vom waußengeleitetcn " einzclncn" oder von einem mpostmodernena"s Individuum gesprochen wird, gemeint ist im einen wie im anderen Fall cin Artefakt. Der einzelne ist nur noch das Produkt einer Synthese vorgegebener Eigenschaften. ${ }^{96}$ Alle auf eín uniformes, generell festgelegre Erwartungen reproduxicrendes Verhalten abziełenden Bestrebungen sind insofern bewußt gegen eine Individuierung aufgericheete Barrieren. Nicht anders verläult übrigens die Entwicklung dort, wo korporatistischen Strukturen der Vorzug gegeben wird. Ebensowenig wie etwa staatliche Ämter sind Korporationen Agenturen des cinzelncn. Genauso wie die staatliche Administration erhebe vielmchr dic Korporation den Anspruch auf ein ihren Erwartungen konformes Verhalten. Je mehr sich aber die Korporation als der eigentliche Trägcr aller Meinungsbildung in der Gesellschaft versteht, desto deurlicher nimme auch der Druck auf den einzelnen zu, sich als Teil der Korporation zu sehen und sein gesamtes Verhaiten dementsprechend einzurichten. Mag deshalb sein, daß die Etablierung korporatistischer Strukturen die staatlichen Prärogativen cinschränkt und eine Dezentralisicrung der politischen und ökonomischen Entscheidungsprozesse zur Folge hat. Die Vorherrschaft der Korporationen restiruiert trorzdem nichı die individuelle Entscheidungs- und Handlungsfäligkeir. Das Entscheidungszentrum verlagert sich lediglich auf kollekrive Einheiten, die in der Indjvidualität des einzelnen sowohl ihre interne Ordnung als auch ihre Arrangcments untereinander destabilisiercnden Faktor erblicken.

Die Kritik an den Kolonialisierungstendenzen führt freilich keineswegs zwangsläufig dazu, den Zusammenhang zwischen der Individuierung des einzelnen und seiner Vergesellschaftung ${ }^{97}$ zu verlcugnen. Selbstreflexion serzt zwar ein Mindestmaß an Distanz und damit auch ein Minumum an Indifferenz gegenübcr dem einzelnen voraus. Nur solange der gerade durch die Indifferenz gesicherte Abstand gewährleister ist, hat der einzclne cine Chance, sich mit der eigenen Lage ebenso wie mit den eigenen Handlungsmöglichkeiten wirklich selbst auseinanderzusetzen. Der Individuierungsprozcß kann sich deshalb erst vor dem Hintergrund cincr dic Gesellschaft aufspaltenden Atomisicrung vollziehen. In diesem, allerdings auch nur in diesem Sinn hat die Nationalversammlung mit der Loi le Chapelier genauso wie mit den ihr vorausgehenden Entscheidungen den citoyen keineswegs olne Rücksichr, ja auf Kosten des homme social konstituiert. ${ }^{88}$ Die Vereinzelung löst jedoch den einzelnen nicht aus der Gesellschaft heraus, sic ist vielmehr condicio sine qua non seiner sozialen Integration, weil sic es ihm ermöglicht, die ejgene Lebensgeschichte als einen sich in der Gesellschafic abspielenden Vorgang wahrzunchmen, mithin als Ergcbnis konstanter sozialer Interaktion. Kurzum, die Vereinzclung ist sowohl Barriere gegen einc den einzelnen kolonialisierende Okkupation seiner Lebenswelt als auch Grundlage aller sich auf eine selbständige Meinungsbildung gründenden,

9: Habermas, Theorie des kommunikauven Handelos 2 (1981) unsb. s22 ff.

93 Vgl. Simini5, is Univ. Penn. L.R. 71 yff. (1987), in Verrechelichung. a. a. O., i 66 ff.

94 Riesman, Dic consame Masse (1958) i37ff., 25 iff.

95 Baudrillard. Magazıne litcérarc Heft 264 (April 1989), 19.

96 Wolsh am deutlichsıcn komm dies be Baudrilard, a. a. O., 20I. zun Ausóruck.

97. Dazu vor allem Habernax, Nachmenphysisches Denken (1988) 179 If. $223 \mathrm{ff}$.

9\$ Vigl. freslicis Fures, Révolueıon, 1.3.0., 87 f 
den Konnex zur Gescllschaft stcus berücksichtigenden Partiziparion an den sozialen Entscheidungsprozessen. Das eigentliche Problem liegt daher nicht in der Einsicht in die Verknüpfung von Individuierung und Vergesellschaftung, sondcrn in der Fähigkeit, die Einsamkeit zu ertragen und in der Aufgabe, die Garantien der Vereinzelung sowie die Modalicäten der Vergesellschaftung so zu gestalcen, daß es zu einem den einzelnen als Subjekr begreifenden und respekcierenden Individuierungsprozeß kommen kann. So gesehen, ist die Loi le Chapelier keineswegs nur eine längst abgeschlossene Etappe in dor Geschichte einer gescheiterten Utopie. Sie zwingt vielmehr racl wie vor dazu, sich mit den Konsequenzen jeder Regelung auseinanderzusctzen, die dem einzelnen direkr oder indirekt die Subjekreigenschaft abspricht und erinnerc zugleich daran, daß nur solange der einzelne auch Subjekr bleibt, das Individuum eine Chance hat, mehr zu sein, als ein wherumgeisicrndes Phantom «. 99 